\title{
Una reconsideración de las perspectivas económicas de México ${ }^{1}$
}

Julio López G. $^{2}$

El pobre desempeño económico de México en las últimas dos décadas, y la baja de la tasa de acumulación de capital, han dañado seriamente sus perspectivas de crecimiento futuro. No obstante, el panorama para este país no tiene por qué ser necesariamente adverso, y en este artículo discuto por qué hay razones para el optimismo si se aplica una estrategia económica que ponga el acento en el crecimiento y el pleno empleo. Obviamente, una propuesta detallada está por encima de las capacidades de un sólo autor, y aquí ofrezco apenas sugerencias generales, referidas a temas macroeconómicos básicos. Muchos lectores se darán cuenta de que la mayoría de las ideas propuestas no son particularmente novedosas; y, lo que hago es sobre todo rescatar y ahondar en conceptos desarrollados por economistas latinoamericanos estructuralistas, revividos recientemente por dos miembros destacados de dicha escuela: "Vivir con lo nuestro" (Aldo Ferrer), y "El desarrollo desde dentro" (Osvaldo Sunkel). En este contexto, pretendo demostrar que:

a) A pesar del estancamiento económico y las bajas tasas de acumulación de capital por más de dos décadas, México tiene abundantes recursos ociosos. Su principal tarea debería ser, por tanto, poner a trabajar dichos recursos ociosos y, en particular, la fuerza de trabajo desempleada y el equipo de capital sin utilizar.

b) Un mayor uso de las capacidades productivas ociosas y de la mano de obra desempleada actuales, puede hacer posible una recuperación económica. Así, México puede lograr reanudar su crecimiento a tasas elevadas en el corto plazo. El principal problema, sin embargo, es cómo llevar esto a cabo, de manera tal que se garantice el equilibrio externo.

c) Un estímulo al tipo de cambio real, acompañado de medidas por el lado de la oferta que beneficien a los productores nacionales puede contribuir, en el corto plazo, a hacer crecer los niveles de producción y empleo, en condiciones de balance externo. Una "devaluación compensada" también puede ayudar a hacer

(1) Este trabajo se pudo realizar gracias al apoyo económico de DGAPA-PAPIIT, a través del Proyecto IN301606. Quiero agradecer por sus sugerencias a A. López, G. Bracho, A. Hofman y Tony Thirlwall por sus comentarios a una versión anterior de este artículo; así como a Fernando C. de Carvalho con quien desarrolle en otro trabajo algunas de las ideas expuestas aquí (López; Carvalho, 2008). Pero mi deuda mayor es con Ignacy Sachs, con quien he discutido a lo largo de muchos años los temas de este artículo. Traducción del inglés de Luis Reyes Ortiz.

(2) Universidad Nacional Autónoma de México. E-mail: gallardo@servidor.unam.mx. 
frente a problemas sociales y económicos, asociados con la depreciación de la moneda.

d) La aceleración del crecimiento en el futuro cercano podría facilitar lograr una alta tasa de crecimiento en el mediano y largo plazos, ya que la mayor demanda y utilización de capacidad traen consigo mayores ganancias, las cuales pueden ser reinvertidas. Sin embargo, una estrategia de crecimiento de mediano y largo plazo implica también una cuidadosa estrategia de inversión.

e) Una estrategia de inversión debe tener tres objetivos. En primer lugar, deben llevarse a cabo esfuerzos para reducir los desembolsos de capital por unidad de producto. Segundo, deben canalizarse inversiones para abrir nuevas posibilidades de exportación y nuevas áreas para sustituir importaciones. Tercero, deben crearse industrias selectas que fomenten el progreso técnico.

El artículo está organizado como sigue. En la próxima sección discuto algunas cuestiones generales. En las secciones dos y tres considero las estrategias de crecimiento de México en el corto y largo plazo, respectivamente. Concluyo el artículo con algunas consideraciones finales de tipo general.

\section{Cuestiones generales}

Cuando la tarea a realizar es concebir y poner en práctica una política económica progresiva en la realidad actual, surgen dos tipos de dificultades. La primera involucra a los obstáculos objetivos, que analizaré detalladamente a lo largo de este trabajo. Sin embargo, en ésta sección, me gustaría centrarme en una segunda dificultad, más sutil que la primera, y que involucra asuntos subjetivos o ideológicos.

El problema es que la puesta en marcha de una estrategia económica progresista no solamente afectaría los intereses de las clases acomodadas de la sociedad; las cuales, naturalmente, se oponen a cualquier intento de limitar sus privilegios políticos y económicos. La dificultad es, también, que el mero diseño de tal estrategia rompería radicalmente con lo que es actualmente el paradigma predominante en economía. Cierto, este paradigma no es para nada robusto. Se basa en la errónea idea teórica de que se puede lograr un óptimo económico (un óptimo de Pareto, para utilizar nuestra jerga de economistas) en un sistema capitalista de libre concurrencia, sin necesidad de recurrir a la intervención estatal. Antes y después de Keynes se han hecho todo tipo de intentos para demostrar esta hipótesis, pero todos ellos han fallado; incluso cuando se hace uso de técnicas matemáticas cada vez refinadas (Ingrao; Israel, 1990). La historia económica tampoco presta apoyo alguno a la idea de que se logren el pleno empleo de los recursos y el rápido crecimiento bajo una economía capitalista sin (o con poca) intervención estatal (Korpi, 2002). 
Sin embargo, a pesar de carecer de bases teóricas o históricas sólidas, el paradigma en cuestión se ha reinstalado, en una especie de "restauración de los Borbones", en las ideas y la visión de la mayoría de los economistas y políticos, así como del común de la gente, y es considerado hoy un tipo de verdad revelada y absoluta. La aceptación incondicional del paradigma es una de las razones de por qué las propuestas hechas por partidos políticos o grupos progresistas, por lo general, no son muy diferentes de aquéllas formuladas por partidos o grupos conservadores.

En el pasado, no obstante, aquéllos de nosotros en el campo progresista compartíamos ideas básicas que nos separaban radicalmente del campo conservador. Una de ellas sostenía que la sociedad, a través del Estado, puede y debe diseñar mecanismos y aplicar políticas que garanticen el pleno empleo y mejoren las condiciones de vida para la mayoría. En verdad, la noción de que la intervención estatal en la economía es indispensable para corregir las fallas del mercado y para promover el crecimiento no es un patrimonio exclusivo de la izquierda. Estas ideas fueron aceptadas desde fines de la Segunda Guerra Mundial, y hasta aproximadamente mediados de la década de los setenta, aunque a veces de mala gana, incluso por partidos conservadores. Tanto así, que la intervención estatal, bajo gobiernos con ideologías diferentes, hizo posible que países capitalistas desarrollados crecieran mucho más rápido de lo que habían crecido anteriormente a lo largo de su historia. ${ }^{3}$

Algo parecido a lo que sucedió con países capitalistas avanzados pasó en México. ${ }^{4}$ El llamado "milagro Mexicano" de los años cincuenta y sesenta, cuando el PIB por habitante creció a una tasa anual de casi 3.5 por ciento (con un aumento más de $50 \%$ en 15 años), no estuvo basado en capitalismo de competencia perfecta, sino que incluyó una significativa dosis de intervencionismo estatal (Guerrero, 2005; Suárez, 2005; Gracida, 2005). El cambio de trayectoria, comenzado a mediados de los ochenta, trajo consigo un deterioro del desempeño

(3) Con el advenimiento del "Estado de bienestar" se logró una importante reducción del desempleo y una gran mejora del nivel de vida de las mayorías. Dicha "reforma crucial" (para usar el término apto acuñado por Kalecki y Kowalik, 1971[1991]) fue un factor clave que contribuyó a que se concibiera al socialismo como posible gracias a una evolución gradual, basada en el desarrollo y transformación del capitalismo mismo. Las razones por las que las medidas de administración de la demanda fueron abandonadas en países capitalistas avanzados son magistralmente discutidas por Korpi (2002).

(4) Muchos gobiernos latinoamericanos intervinieron decididamente en la economía para hacer frente a la crisis mundial de los años treinta. Sin embargo, hasta donde sé, el primer plan coherente en estas líneas fue propuesto (aunque no puesto en marcha) en Argentina a finales de 1933, curiosamente bajo un gobierno de derecha; y fue ideado por Raúl Prebisch (Prebisch 1991-1993, v. II, ch. 92). Celso Furtado explica con su agudeza característica cómo la compra del superávit de café por parte del gobierno de Brasil implicó una expansión del gasto deficitario; lo que mantuvo la demanda agregada e interna, así como el empleo. En sus palabras, "El valor del producto [café] destruido fue mucho menos del valor de su ingreso creado. De hecho, estábamos construyendo las famosas pirámides de Keynes" (Furtado, 1962, p. 198).

Economia e Sociedade, Campinas, v. 17, número especial, p. 677-694, dez. 2008. 
económico. El PIB por habitante se ha mantenido estancado durante todo el periodo en el cual la estrategia, denominada "neoliberal" a falta de un mejor término, se inició y se consolidó.

El cambio de estrategia que se necesita en México actualmente requiere que el Estado recupere un papel activo, y que la política económica puesta en marcha tenga como objetivos asegurar la plena utilización de los recursos, la acumulación del capital necesario para el crecimiento sostenido de largo plazo y los equilibrios interno y externo. En el resto de las páginas de este documento formularé algunas ideas al respecto.

\section{Discutiendo el corto plazo}

El potencial de crecimiento de corto plazo de México es aún importante. La baja utilización de una parte de su capacidad productiva, y de mano de obra, es prueba de su potencial. Además, toda evidencia disponible muestra que hay factores de la producción que permanecen sin utilizarse, incluso en el punto más alto del ciclo económico, lo que refleja un defecto sistémico y no una situación coyuntural.

Primero, se sabe que el desempleo de mano de obra es muy amplio. Cierto, las tasas de desempleo abiertas son bajas (fluctuando entre 4 ó 5 por ciento del total de la mano de obra). Pero el desempleo disfrazado es alto. Algunas estimaciones mencionan tasas apenas inferiores a 40 por ciento de la Población Económicamente Activa. El desempleo sería mucho mayor si México no tuviera la válvula de escape de la emigración a los Estados Unidos (alrededor de un millón de trabajadores por año; i.e. cerca de 1.5 por ciento de su fuerza de trabajo). Finalmente, nótese que no es el caso que los desempleados no tengan la habilidad suficiente para obtener un trabajo. De hecho, los trabajadores calificados y los no calificados están representados casi en la misma proporción entre los desempleados.

Segundo, aunque difícil de encontrar, y algo debatible en el terreno metodológico, la información disponible sugiere que un gran porcentaje de la capacidad productiva permanece hoy en día ocioso. Una importante evidencia proviene de la respuesta de administradores de empresas manufactureras a encuestas. De acuerdo con las encuestas de INEGI, a fines de 2007 las empresas utilizaron alrededor del 82 por ciento de su capacidad, y dicha tasa nunca excedió el 85 por ciento en el periodo que va de 1996 a 2007.

México se beneficiaría si utilizase la capacidad productiva ociosa, ya que el costo de oportunidad de producir con la capacidad productiva ociosa de hoy en día está, en general, por debajo del precio internacional de los productos involucrados (López; Huerta, 2006). En otras palabras, es más provechoso utilizar 
dicha capacidad para exportar o sustituir importaciones, incluso si esto requiriese subsidiar a los productores internos, que dejarla sin utilizar. Además, la tasa de capacidad ociosa proporciona una medida aproximada de cuánto pudiera aumentar la producción. No siempre sería fácil ampliar la producción, ni podría el país lograr esto en un día o dos, pero no es imposible. Pero, entonces, ¿cómo podría lograr esto México? Esta es la pregunta que trataremos de responder a continuación.

Promover una expansión económica de corto plazo requiere, en primer lugar, de un incremento de la demanda acompañado de una política crediticia expansiva selectiva. La mayor demanda y las mayores ventas aumentan el empleo, los salarios y las ganancias. La disponibilidad de crédito en abundancia y barato a los productores internos contribuiría a una alta elasticidad de la oferta. Las mayores ganancias mejoran las expectativas y los ahorros propios de las empresas, estimulando la inversión.

Sin embargo, una mayor producción requiere también de mayores importaciones. En consecuencia, para mantener el balance externo, un segundo requisito de la estrategia de recuperación del crecimiento es dedicar esfuerzos adicionales para mejorar la competitividad de la producción interna.

La competitividad puede mejorar con un mayor tipo de cambio real; sin embargo una gran depreciación de la moneda, lo cual es una forma de elevar el tipo de cambio real en el corto plazo, también genera problemas. En particular, ella puede afectar negativamente el consumo y la inversión, además de que contribuye al aumento de la inflación. Alguna evidencia muestra que los efectos negativos en la demanda interna no son, por lo general, compensados por la mejora de la balanza comercial, generada por la depreciación.

Si descartamos una gran devaluación, ¿cómo puede México lograr un aumento lo suficientemente grande del tipo de cambio, y de su competitividad? Hay alternativas, todas basadas en la noción de la "devaluación compensada", propuesta hace algún tiempo por dos economistas latinoamericanos (Schydlowsky, 1967; Diamand, 1973).

Una posibilidad sería subsidiar la producción interna. Los subsidios podrían tomar la forma de exenciones a las empresas de las cargas impositivas asociadas a la nómina salarial. Naturalmente, el gobierno - en colaboración con representantes del sector privado - debería asegurar que se transfieran a los consumidores los menores costos, evitando que ello signifique simplemente un aumento de los márgenes de ganancia. Para evitar ir en contra de las reglas del GATT, el Estado podría otorgar subsidios sólo a la producción para la venta en el mercado interno, como Keynes propuso alguna vez (Keynes, 1943). 
Podrían lograrse resultados análogos mediante una depreciación de la moneda compensada con un descuento al Impuesto al Valor Agregado para los bienes de consumo básico, o compensada con subsidios a los grupos de bajos ingresos. De manera alternativa, el Banco Central podría poner en su lugar un sistema dual de tipo de cambio, donde a los exportadores se les pague una tasa mayor, y a los insumos básicos para exportación se les da un tipo de cambio preferencial.

Existen otras alternativas para lograr un tipo de cambio competitivo, al mismo tiempo que se asegura la estabilidad de los precios y de los salarios reales. De hecho, las experiencias históricas de México y de Latinoamérica son ricas en alternativas, y debemos estudiarlas más a conciencia de lo que normalmente se hace. La elección de una u otra de dichas alternativas dependería de muchos factores, tales como sus efectos relativos en el consumo masivo, sus requisitos administrativos y las circunstancias del país en el momento.

De cualquier forma, la posibilidad de establecer y de mantener un tipo de cambio a una tasa competitiva requerirá de algún grado de control de los movimientos de capital (Bhaduri y Matzner, 1990; Steindl, 1990; Frenkel 2006). Es cierto que si el país tiene una balanza externa equilibrada es menos probable que quede expuesto a los vaivenes de los movimientos de capital especulativo. Pero no debemos tampoco olvidar que los mercados financieros internacionales son muy volátiles, y que los choques externos pueden tener consecuencias desastrosas. A propósito, México tuvo una corta experiencia con controles de capital a principios de la década de los ochenta. Funcionó mientras estuvo en vigor, y se abandonó sólo porque el gobierno cambió y la administración entrante estaba en contra de dicha medida, en tanto que también las organizaciones financieras internacionales las combatían de manera radical.

\section{El crecimiento de mediano y largo plazo}

En los párrafos anteriores nos referimos al corto plazo, y ahora consideraremos las necesidades de una estrategia de mediano y largo plazos. Iniciamos la discusión con los aspectos más básicos del crecimiento, aquellos relacionados con el lado de la oferta, y para llevar a cabo la exposición hago uso de dos modelos simples y bien conocidos.

El primero se refiere al crecimiento de la capacidad productiva potencial, y la versión que utilizaremos fue propuesta por Kalecki $(1969,1970)$. Sean $\mathrm{r}^{\mathrm{K}}$ la tasa de crecimiento de la capacidad productiva potencial, i la tasa de inversión (bruta), у к la relación técnica capital-producto (esto es, el número de unidades que se requieren para, efectivamente, producir una unidad). Sea d la tasa de depreciación, y u la tasa anual extra de crecimiento de la producción potencial que 
un país podría obtener si utiliza mayor y mejor capacidad productiva existente (nos referimos a i, u y d como proporciones del PIB). Especificamos la tasa de crecimiento de la capacidad productiva como:

$$
r^{k}=\frac{i}{\kappa}+u-d
$$

Esta sencilla fórmula muestra que, dados $\mathbf{k}, \mathbf{u}$ y $\mathbf{d}$, la capacidad productiva potencial puede aumentar sólo si la tasa de la inversión i aumenta. Sin embargo, nótese que $\mathbf{k}$, $\mathbf{u}$ y $\mathbf{d}$ son parámetros que un país puede modificar, hasta cierto punto, con medidas de política económica. De esta manera, puede aumentar u utilizando más (y mejor) la capacidad productiva. Asimismo, al alargar el tiempo de vida de la capacidad productiva podremos reducir d. Finalmente, se puede reducir la relación capital-producto $\mathbf{\kappa}$, recurriendo a técnicas más intensivas en mano de obra cuando ello sea posible.

El segundo modelo está basado en Prebisch (1951), y fue revivido más recientemente por Thirlwall (1979). Supongamos una situación inicial con una balanza comercial en equilibrio. Prebisch y Thirlwall demostraron que, bajo ciertos supuestos y, en particular, suponiendo un tipo de cambio real dado y haciendo abstracción de los flujos de capital, la tasa de crecimiento del producto compatible con el equilibrio externo $\mathbf{r}^{\mathbf{x}}$ se puede expresar como:

$$
r^{x}=\frac{x}{\pi}
$$

Donde $\mathbf{x}$ es la tasa de crecimiento de las exportaciones, y $\boldsymbol{\pi}$ es la elasticidad ingreso de las importaciones. La evidencia empírica sugiere, primero, que a pesar de que un país puede usar el tipo de cambio como un instrumento de política, no puede hacerlo recurrentemente. Y, segundo, muestra también que muy pocos países pueden mantener un constante superávit de importaciones, de manera que, en el largo plazo, las importaciones tienden a financiarse a través de los ingresos obtenidos con las exportaciones. Consecuencia de lo anterior es que, generalmente, la restricción sobre el crecimiento del producto es la tasa de crecimiento de la capacidad para importar, misma que está determinada por la tasa de crecimiento de las exportaciones y por la elasticidad ingreso de las importaciones, tal como en la ecuación (2).

Ahora bien, en base a la ecuación (1), propondría que la estrategia de inversión de México debe ser dar prioridad, en primer lugar, a la inversión indispensable para hacer posible la más completa y eficiente utilización de la capacidad productiva heredada. En verdad, hay muchos casos en que el desempleo de la capacidad productiva se debe a cuellos de botella a nivel empresarial o a nivel macro, que dificultan la completa o eficiente utilización del capital. En 
determinadas circunstancias para corregir los problemas se requerirán inversiones, o reformas organizativas, o de ambas, que implican un mayor tiempo de gestación. Por ende, un uso más completo de la capacidad productiva requiere también de inversiones. Asimismo, por las razones ya discutidas, la estrategia de inversión debe privilegiar aquella necesaria para alargar el tiempo de vida de la capacidad productiva, y aquella intensiva en trabajo y que tiene una baja relación capitalproducto.

Se deduce del punto anterior que México podría lograr un ritmo de crecimiento de largo plazo satisfactorio con un aumento relativamente pausado de la tasa de inversión. No quiere decir esto que la tasa de inversión no se deba aumentar; sino más bien que no se necesita aquí proponerse una tasa de inversión excesiva.

Los pioneros de la economía del desarrollo (Rosenstein-Rodan, 1943; Mandelbaum, 1945; Prebisch, 1951; Furtado, 1953) favorecieron este enfoque. Sin embargo, notemos que esta propuesta difiere de la promovida por la literatura de estrategia industrial hoy predominante; que denominaré en lo que sigue estrategia "modernizadora". Ésta última da preferencia a un drástico aumento de la tasa de inversión, y a la rápida renovación de la capacidad productiva y a la inversión en actividades nuevas y técnicamente sofisticadas. En cambio, aquí propongo un aumento pausado de la tasa de inversión, priorizando las inversiones con técnicas de baja intensidad de capital, así como las inversiones complementarias que son necesarias para utilizar al máximo la capacidad productiva antigua.

Los defensores de una estrategia "modernizadora", probablemente criticarían el enfoque que aquí proponemos, argumentando que México necesita acelerar el progreso técnico y el ritmo de crecimiento de la productividad del trabajo. Probablemente agregarían que una mayor productividad del trabajo significa menores costos, y hace a la producción nacional más competitiva; lo cual es necesario para mantener la balanza comercial equilibrada.

Más adelante matizaré algo la opción de ahorro de capital que aquí favorezco, pero antes quiero discutir brevemente el enfoque de modernización rápida para un país como México. Creo que los proponentes de la estrategia "modernizadora" pierden de vista algunos temas importantes. Por un lado, no consideran que esa estrategia implicaría mayores costos. Dichos costos vienen en dos formas. Primero, implicaría una mayor tasa de inversión, y una menor tasa de consumo, necesaria para lograr la misma tasa de crecimiento del producto. Pero una tasa demasiado alta - del orden del 30 por ciento o del 40 por ciento, como en China por ejemplo - puede exigir tales sacrificios a las generaciones presentes que el mismo podría no ser viable con métodos democráticos. Segundo, esa estrategia estaría asociada a una mayor propensión a importar, ya que las líneas modernas de 
producción y de tecnología sofisticada tienen un mayor contenido de importaciones.

Por otro lado, los proponentes de la rápida modernización tampoco parecen sacar las implicaciones del hecho que la competitividad no depende directamente de la productividad, sino de los costos. Consideremos el caso desde el punto de vista de la economía como un todo. Aquí, lo que importa son los costos de oportunidad de los factores; esto es, cuánto perdemos en la producción si quitamos al factor $\mathrm{x}$ de donde éste es menos eficiente. Cuando un país tiene recursos ociosos, su costo de oportunidad es cero. La justificación de un tipo de cambio más alto, o de subsidios a la exportación o a la substitución de importaciones, es precisamente acercar los costos y precios privados a los costos y precios sociales, para orientar la inversión y el uso de los recursos de acuerdo a la disponibilidad de factores.

Más aún, el énfasis aquí recomendado no dificultaría, sino que estimulará la inversión, el progreso técnico, y la modernización. Con una tasa de inversión en aumento, pero creciendo modestamente México lograría mayores niveles de producción, empleo, salarios y ganancias en el corto plazo. Los niveles de empleo y consumo, así como las ganancias, serían mayores en una etapa inicial. Las mayores ganancias estimularían mayores inversiones. Por el otro lado, una parte del incremento del consumo iría a mayores gastos en educación. El mayor empleo y el mayor gasto en educación propiciarían una calificación y entrenamiento más veloz de la fuerza de trabajo.

Reconozco que, desde cierto punto de vista, el énfasis de esta estrategia implica disminuir la modernización. Pero aquí, el argumento postulado hace tiempo por Kalecki (en su importante, pero poco conocido, estudio de la economía israelí), sigue siendo válido:

La inversión en ramas de la industria donde todavía existe capacidad sin utilizar, a pesar de que el proceso implicaría una modernización considerable, es un lujo que, por ahora, no puede permitirse la economía. Este es especialmente el caso, ya que... [en Israel]...no hay escasez de fuera de trabajo en este momento y, por lo tanto, esta forma de modernización, cuyo objetivo sería ahorrar trabajo, no tiene mucho sentido desde el punto de vista de la economía como un todo. Podría argumentarse que la modernización es necesaria para reducir costos y, por ende, incrementar la capacidad para competir en el exterior, lo que aumentaría las exportaciones. Sin embargo, desde el punto de vista del ahorro de moneda extranjera, el cual es el factor más escaso en la economía israelí, es mucho más razonable pagar primas de exportación, sin importar qué tan poco natural parezca tal forma de subsidio a métodos de producción obsoletos (Kalecki, 1951, p. 103, traducción propia).

Sin embargo, de acuerdo con el modelo Prebisch-Thirlwall (ecuación 2), un ritmo alto de crecimiento del producto sólo es sostenible si va acompañado con 
un fuerte aumento del ritmo de crecimiento de las exportaciones, y con una baja elasticidad ingreso de las importaciones. Ello plantea un segundo requerimiento de la estrategia de largo plazo, el cual es asegurar un crecimiento acelerado de la inversión tanto en las industrias exportadores, como en aquellas capaces de sustituir importaciones.

En este contexto, no está de más recordar que la experiencia histórica de sustitución de importaciones ha sido muy criticada, y que la sustitución de importaciones misma se dejó casi totalmente de lado en la experiencia reciente mexicana, en contraste con lo sucedido con el estímulo a las exportaciones. Así fue como México logró tasas de crecimiento de exportaciones fenomenales; pero esto ocurrió junto con un drástico aumento de la elasticidad de la demanda de importaciones, el cual estuvo, en gran medida, motivado por la creciente necesidad de insumos importados necesarios para las exportaciones. ${ }^{5}$ El aumento de la elasticidad de importaciones es, por su parte, la razón principal de por qué la alta tasa de crecimiento de las exportaciones no ha sido acompañada por una alta tasa de crecimiento del producto.

Podríamos comparar las diferentes experiencias de México y China en este aspecto. Entre 1980 y 1997 la participación de las exportaciones manufactureras de México en el total de exportaciones manufactureras mundiales creció 2.0 puntos porcentuales, pero su participación en el valor de la manufactura cayó 0.7 puntos porcentuales. En contraste, en China los cambios fueron de 2.7 y 2.05 , respectivamente.

Un tipo de cambio real competitivo y estable es fundamental para impulsar la canalización de inversiones hacia los sectores de bienes transables; y por ello concuerdo con la afirmación de Frenkel (2006) de que la preservación de un tipo de cambio real competitivo y estable (TCRCE) es de las mejores contribuciones que puede hacer la política macroeconómica al crecimiento y al empleo. Es verdad que la moneda nacional no puede depreciarse continuamente; sin embargo, un tipo de cambio real competitivo y estable estimula las inversiones hacia las industrias exportadoras y sustituidoras de importaciones. Esto último, a su vez, aumenta la capacidad productiva y la productividad del trabajo, mejorando, así, las condiciones de las ramas de oferta de exportaciones y de competencia de importaciones.

Esta es, nuevamente, una importante lección de la experiencia China (y de los países del este de Asia, en general), donde las altas tasas de crecimiento de las

(5) El patrón de exportaciones de México tiene dos características negativas adicionales. Por un lado, las exportaciones tan sólo vienen de un reducido número de empresas tecnológicamente avanzadas, siendo la mayoría ramas de transnacionales. Por otro lado, las exportaciones están fuertemente sesgadas a favor de los bienes de "alta" tecnología e intensivos en capital, y en muchos casos el proceso interno de producción está limitada al ensamblaje de partes y piezas importadas. 
exportaciones no se lograron depreciando continuamente la moneda, sino a través de continuas ganancias de competitividad en el sector de bienes transables.

Agregaría, sin embargo, dos observaciones al planteamiento recién citado de Frenkel. En primer lugar, el tipo de cambio competitivo no es siempre expansionário desde el punto de vista de la demanda. Por ejemplo, en México la información disponible, y estudios del presente autor, sugieren que un tipo de cambio alto puede tener efectos contraccionistas sobre la demanda. En segundo lugar, hay instrumentos más poderosos y eficaces para orientar la inversión hacia el sector productor de bienes transables. Por ejemplo, los créditos preferenciales, y los convenios para efectuar inversiones de capital mixto privado-público pueden ser fundamentales o indispensable. En este sentido, sería muy importante recuperar la experiencia pasada de México, donde el gobierno conjuntamente con el sector privado, planificaban las prioridades de inversión tomando en cuenta la demanda interna e internacional futuras, y creaban empresas de capital mixto para establecer industrias claves en las actividades correspondientes.

Por ultimo, pero no menos importante, la estrategia de inversiones debe cimentar el camino para que el país adquiera capacidades tecnológicas autónomas. Parece ser que hay algunas industrias particulares, a veces muy intensivas en capital, que pueden incentivar el progreso técnico. El último está limitado cuando dichas industrias generadoras de tecnología están ausentes, y el país corre el riesgo de perder bases para el desarrollo tecnológico autónomo. Frecuentemente toma tiempo que maduren dichas industrias. Esta es que hace necesario iniciarlas en etapas de la industrialización relativamente tempranas. Esto no parece ser tarea imposible para un país de un nivel medio de desarrollo. Debemos recordar que Corea y Taiwán, por ejemplo, no tuvieron éxito en fundar algunas industrias de tecnología avanzada en etapas tempranas de su desarrollo.

Consideremos ahora los aspectos de demanda en una estrategia de largo plazo. Partiré con un par de afirmaciones muy básicas. La primera es que los tres principales impulsores del crecimiento por el lado de la demanda deberán ser la inversión privada, el gasto público, y el consumo de los sectores de bajos ingresos. La segunda afirmación está muy relacionada con la anterior. Se trata de que en una economía moderna el estado puede, y debe, modular la demanda agregada usando de manera combinada las políticas fiscal y monetaria; y que su capacidad de hacerlo se amplía si está dispuesto a usar controles administrativos, que en las economías periféricas son más necesarios que en aquellas desarrolladas. En verdad, sostener que el estado puede modular la demanda sería una afirmación trivial e innecesaria, si no fuera porque en el paradigma teórico hoy dominante algo menos en el quehacer práctico de los gobiernos - esa posibilidad se niega. Aquí al contrario esa posibilidad se reconoce y se valora. Es más, se afirma que el único criterio válido para medir la sostenibilidad de las políticas de demanda, es 
que éstas deben contribuir al pleno aprovechamiento y ampliación de las capacidades productivas, así como al equilibrio externo y de los precios en condiciones de alto crecimiento. Esta afirmación puede parecer demasiado general; pero creo que carece de sentido intentar capturar a través de una fórmula un tema tan complejo.

Me referí antes a la necesidad de elevar - pausadamente - la tasa de inversión y ahora me ocuparé de la demanda gubernamental. Hay al menos tres razones que justifican elevar de manera significativa el gasto público. La primera es que la inversión pública puede ser clave para eliminar cuellos de botella que limitan el pleno uso de la capacidad instalada o desestimulan su ampliación. La segunda razón también se mencionó antes; se trata de que el gasto público productivo debería contribuir a la corrección de las distorsiones del mercado por caminos complementarios a la modificación de los precios clave de la economía. Por ejemplo, los créditos preferenciales o los subsidios a la producción nacional ayudarían a mejorar la competitividad de nuestras exportaciones y de los substitutos de las importaciones. También, los subsidios y mecanismos focalizados suelen ser más eficaces para dar apoyo productivo a ciertos sectores, como por ejemplo los empresarios informales. ${ }^{6}$ La tercera razón es que en México el gasto público en servicios básicos a la población, como salud, educación, por ejemplo, es sumamente reducido y es indispensable corregir esa distorsión ${ }^{7}$.

Ahora bien, conviene dejar en claro que si bien el gasto público material debe aumentar, el pago de intereses sobre la deuda del estado debería reducirse por la vía de una drástica rebaja de la tasa de interés. En la actualidad ese pago representa en México alrededor del 16 por ciento del gasto del gobierno y 4 por ciento del PIB. De manera que hay mucha tela de donde cortar antes de acercarse siquiera a la "eutanasia del rentista" que Keynes proponía.

De cualquier forma, surge aquí la pregunta de cómo financiar un gasto público en ascenso. En este contexto resulta útil tomar como punto de partida un artículo clásico de Kalecki sobre políticas económicas en economías de capitalismo avanzado. Kalecki consideró tres vías mediante las cuales podría lograrse un crecimiento de la demanda suficiente como para asegurar el pleno empleo tanto de la fuerza de trabajo como del equipo de capital. La primera vía es el gasto público deficitario, la segunda es el estímulo a la inversión privada, y la tercera vía es redistribuir el ingreso en favor de los grupos de ingresos bajos.

(6) Por ejemplo, durante muchos años los gobiernos mexicanos han diseñado un conjunto de políticas de apoyo a la industria bastante sofisticadas y que en el papel se ven muy bien. Pero los fondos públicos para llevarlas a la práctica han sido muy escuálidos.

(7) Entre 1990 y 2004 el gasto público total representó en México, en promedio, poco menos del $18 \%$ del producto. En Brasil el gasto público representa cerca del 36\% del GDP. En los países de la OCDE el porcentaje correspondiente fue 35.4, y en Francia fue $42.6 \%$ (Cepal, 1998). 
En ese trabajo ya citado y en varios otros Kalecki demostró que el gasto deficitario contribuye a elevar la demanda y el empleo; y que el mismo no generará inflación si hay capacidades ociosas amplias, y que no elevará la tasa de interés si va acompañado de una política monetaria activa. ${ }^{8}$ Sin embargo, él se mostró más bien partidario de financiar el mayor gasto público con impuestos a los sectores de altos ingresos. Tal preferencia no la justificaba por un supuesto impacto inflacionario mayor del déficit, ni tampoco por una eventual necesidad del estado de obtener dinero del sector privado. Ella se fundamentaba en la necesidad de limitar la expansión del consumo de los sectores ricos de la población. En México, donde la concentración de la renta es excesiva y el consumo suntuario enorme, esa opción de gravar a los grupos de altos ingresos es ciertamente muy válida. Por lo de más, si los impuestos recaen sobre las ganancias de las firmas, ellos no necesariamente restarán estímulos a la inversión privada, por cuanto el mayor gasto gubernamental y las ventas más altas, compensan en parte la caída de la ganancia unitaria neta de impuestos (Kalecki, 1990 [1937]).

Por último, respecto del crecimiento del consumo privado de los sectores de bajos ingresos, éste debería verse estimulado en primer término por el crecimiento del empleo asalariado; y también por un esfuerzo específico de redistribución del ingreso a favor de los más pobres.

Sin embargo, en el contexto de México, la redistribución del ingreso por la vía de alzas salariales generalizadas no parece ser la mejor solución. Esas alzas se transmiten normalmente a los precios y ello tiene dos efectos perversos. Por un lado, el alza de los precios dificulta mantener un tipo de cambio competitivo y estable. Por el otro, cuando los mayores salarios se traspasan a los precios, se perjudican los trabajadores más pobres, que en México no son asalariados en empresas formales sino campesinos sin tierra o con poca tierra, trabajadores por cuenta propia o empleados en establecimientos informales (López, 2005). Para evitar las alzas de precios habría que controlarlos o subsidiar a los bienes básicos; y estas medidas en general son difíciles de implementar, tanto por razones técnicas y administrativas como sobre todo políticas. Es por lo anterior que nos parece más apropiada una medida más focalizada. Se trata del aumento del salario mínimo, y en especial el agrícola, ya que en México el primero ha caído en términos reales alrededor de 70 por ciento entre 1980 y 2005. El aumentos del salario mínimo tiene la doble ventaja que, por un lado, favorecería a los más pobres de la población, y por el otro no se transmite a los precios. ${ }^{9}$

(8) Por cierto, esta afirmación de Kalecki es similar, y fue expuesta de manera contemporánea a la noción de "finanzas funcionales" de Abba Lerner (1943)

(9) En México, el aumento del salario mínimo tiene un impacto relativamente pequeño sobre el salario promedio; con una elasticidad del segundo del 10\% respecto del primero (López, 1999) 
Para concluir, creo que es importante precisar que aunque es cierto que México puede proponerse alcanzar en el futuro tasas de crecimiento altas, hay también límites de aquellas que será posible alcanzar y sostener. Esos límites tienen que ver con las posibilidades objetivas de conservar el equilibrio externo, por un lado, y la estabilidad de precios, por el otro. Cuando el crecimiento se fuerza demasiado, surgen desequilibrios, sobre todo porque la oferta interna presenta cuellos de botella en diversos sectores o ramas. Esos desequilibrios conducen, tarde o temprano, a la adopción de políticas contraccionistas; o bien son de muy difícil solución bajo un régimen democrático. Es mejor proponerse una tasa de crecimiento relativamente alta y estable, que una tasa muy alta pero insostenible.

\section{Comentarios finales}

He discutido una serie de medidas de política económica que le permitirían a México acelerar el crecimiento en el corto plazo, gracias al pleno y más eficiente uso de la capacidad productiva y de la mano de obra desempleada. Dichas medidas implican una expansión selectiva de la demanda y del crédito a los productores internos, así como mecanismos basados en la noción de "devaluación compensada" que mejoren la competitividad y que mantengan el equilibrio externo cuando se recupere el crecimiento. También sugiero que en su estrategia de largo plazo, debe darse preferencia a la inversión intensiva en trabajo y a inversiones para utilizar más plenamente la capacidad productiva, así como también a aquellas que sirvan para alargar la vida útil de éste. Dicha estrategia implicará, muy probablemente, una baja en (la tasa de crecimiento de) la productividad del trabajo; pero parece una opción adecuado en un país donde las reservas de fuerza de trabajo son muy grandes. Sin embargo, la estrategia de largo plazo también debe contemplar nuevas inversiones al sector de bienes transables, para asegurar altas tasas de crecimiento de las exportaciones, así como la sustitución eficiente de importaciones que haga posible mantener controlada la elasticidad ingreso de las importaciones. Esto es un requisito para preservar la balanza comercial en equilibrio en condiciones de altas tasas de crecimiento del producto. Por último, pero no menos importante, también deberían canalizarse nuevas inversiones hacia sectores y actividades que ayuden a desarrollar las capacidades tecnológicas autónomas.

Finalmente, he señalado que las fuentes de demanda habrán de ser, junto con la inversión privada, el gasto público y el consumo privado, y destacadamente el consumo de los sectores más pobres de la población. Este último debería verse favorecido sobre todo gracias a un fuerte aumento del empleo asalariado, y a la redistribución del ingreso, que se materializaría gracias a los aumentos del salario mínimo. 
De cualquier forma, también debemos entender que, sea cual sea el conjunto de medidas concretas adoptadas, una política económica integral para la recuperación y el crecimiento de mediano y largo plazo, sólo puede basarse en un pacto renovado entre los sectores público y privado. Dicho pacto funcionó durante su experiencia de economía mixta, y le permitió lograr fenomenales tasas de crecimiento económico. Sin embargo, las condiciones de hoy en día son más exigentes. En consecuencia, el rápido crecimiento requiere una estrategia cuidadosamente planeada, donde todos los sectores sociales estén involucrados y participen.

La nueva estrategia no puede representar un paso hacia atrás en relación a algunas opciones que México ha tomado durante éstos últimos años. Entre otras cosas, el fortalecimiento del sector privado, así como la cuidadosa supervisión de los equilibrios macroeconómicos - especialmente el balance externo y la estabilidad de los precios - no están abiertos a debate. De hecho, frecuentemente ocurrieron importantes problemas durante la era de intensa intervención estatal. Para listar sólo unos cuantos, mencionamos las presiones inflacionarias, los grandes déficit fiscales, las tasas de interés reales negativas, las empresas públicas poco eficientes, la arbitrariedad en la protección comercial, y las múltiples decisiones micro-económicas dejadas innecesariamente en las manos de las autoridades gubernamentales. Nadie quiere ver estos problemas e ineficiencias repetirse en México. En especial, quienes defendemos una mayor presencia del Estado en la economía debemos rechazar tajantemente los excesos del pasado.

Por tanto, la estrategia que hemos defendido a lo largo de este trabajo, se encuentra en un marco cuya visión reconoce las ventajas de una economía mixta y abierta, y en que se hacen importantes esfuerzos para que la economía nacional sea muy competitiva en los mercados interno e internacional, así como en la que el mercado se combina con la regulación del Estado.

Para concluir, reconocemos que las políticas económicas y la estrategia que aquí proponemos podrían parecer muy radicales en la actualidad. Cierto, ellas no están lejos de nuestra experiencia histórica, y tampoco se distancian de la experiencia de otros países. Sin embargo, los tiempos han cambiado. Una consecuencia negativa importante de más de dos décadas de neoliberalismo en México ha sido su impacto en la opinión pública. No sólo los promotores del llamado "Consenso de Washington", sino incluso la opinión pública, pueden poner en duda una propuesta de estrategia económica diferente de la hegemónica hoy en día. Pero también debemos recordar en este punto el incisivo comentario de Keynes (1963, p. 90). 
$\mathrm{Si}$, en efecto, la opinión pública fuera una cosa inalterable, sería una pérdida de tiempo discutir asuntos públicos. Y, a pesar de que puede ser el principal negocio de los periodistas y de los políticos cerciorarse de sus características efímeras, un escritor debería, en contraste, preocuparse por lo que la opinión pública debiera ser.

Quizás nosotros, "los escritores", podemos hacer una modesta contribución a cambiar el estado de la opinión pública, y hacer menos utópico un cambio radical de la estrategia económica en México.

\section{Bibliografía}

BANCO DE MÉXICO. Indicadores económicos, varios volúmenes.

BHADURI, A.; MATZNER, E. Relaxing the international constraint on full employment. Banca Nazionale del Lavoro Quartely Review, Mar. 1990.

CARVALHO, F.; FERRARI FILHO, F. El presidente Lula da Silva en el primer tercio de su mandato. Investigación Económica, v. 63, n. 249, p. 55-74, 2004.

CASTRO, C.; LORÍA, E.; MENDOZA, M. A. EUDOXIO: Modelo macroeconométrico de la economía mexicana. México: Fac. Economía, UNAM, 1997.

DE SOTO, H. The mystery of capital: why capitalism triumphs in the west and fails everywhere else. New York: Random House, 2000.

DIAMAND, M. Doctrinas económicas, desarrollo e independencia. Buenos Aires: Editorial Paidos, 1973.

FRENKEL, R. An alternative to inflation targeting in Latin America: macroeconomic policies focused on employment. Journal of Post Keynesian Economics, v. 28, n. 4, p. 573-591, 2006.

; ROS, J. Desempleo, políticas macroeconómicas y flexibilidad del mercado laboral. Argentina y México en los noventa. Desarrollo Económico, IDES, Buenos Aires, v. 44, n. 173, p. 33-56, abr./jun. 2004.

FURTADO, C. La formación del capital y el desarrollo económico. El Trimestre Económico, v. 20, n. 77, p. 88-121, 1953.

1962.

La formación económica del Brasil. México: Fondo de Cultura Económica,

GALBRAITH, J. A theory of price control. Cambridge: Harvard University Press, 1952.

GUERRERO, A. El pensamiento económico de México: etapa de definición del Estado Interventor. In: ROMERO, M. E. Historia del pensamiento económico de México. Ciudad de México: Ed. Trillas, 2005.

GRACIDA, E. La década de 1970 en México: Etapa de transición del pensamiento económico. In: ROMERO, M. E. Historia del pensamiento económico de México. Ciudad de México: Ed. Trillas, 2005.

GURRIERI, Adolfo (Ed.). La obra de Prebisch en la CEPAL. México: Fondo de Cultura Económica, 1982. 
HOFMAN, A. Standarized capital stock estimates in Latin America: a 1950- 1994 updates. Processed. Santiago de Chile: Cepal, 1997.

INGRAO, B.; ISRAEL, G. The invisible hand: economic equilibrium in the history of science. Cambridge, Massachusetts: MIT Press, 1990.

KALECKI, M. (1937). A theory of commodity, income and capital taxation. In: COLLECTED works of Michal Kalecki, Edited by J. Osiatynsky. Oxford University Press, 1990. v. I.

(1944). Three ways to full employment. In: COLLECTED works of Michal Kalecki, Edited by J. Osiatynsky. Oxford University Press, 1990. v. I.

(1951). Report on the main current economic problems of Israel. In: COLLECTED works of Michal Kalecki, Ed. by J. Osiatynsky. Oxford University Press, 1993. v. V.

(1969). Introduction to the theory of growth in a socialist economy. In: COLLECTED works of Michal Kalecki, Edited by J. Osiatynsky. Oxford University Press, 1993. v. IV.

(1970). Theories of growth in different social systems. In: COLLECTED works of Michal Kalecki, Edited by J. Osiatynsky. Oxford University Press, 1993. v. IV.

KEYNES, J. M. (1943). Proposal for an International Clearing Union. In: THE COLLECTED writings of John Maynard Keynes, 25, Ed. by D. Moggridge. London: Macmillan, 1980.

Essays in persuation. New York: W. W. Norton \& Company, 1963.

KORPI, W. The great through in unemployment: a long-term view of unemployment, inflation, strikes, and the profit/wage ratio. Politics \& Society, v. 30, n. 3, p. 365-426, 2002.

KRUGMAN, P.; TAYLOR, L. Contractionary effects of devaluation. Journal of International Economics, Nov. 1978.

LERNER, A. P. Functional finance and the federal debt. Social Research, v. 10, p. 38-51, 1943.

LÓPEZ, J. La macroeconomía de México. El pasado reciente y el futuro posible. Mexico: Editorial Porrua, 1998.

. The macroeconomics of employment and wages in Mexico. Labour, v. 13, n. 4, p. 859-878, 1999.

Income distribution in Latin America. Theoretical considerations and Latin American Experience. Economie Appliquee, Tomo LVIII, n. 3, p. 81-104, 2005.

; HUERTA, R. Restricción externa, ventajas comparativas y crecimiento económico de México (con). Economía. Teoría y Práctica, n. 22, p. 43-62, 2006.

; PUCHET, M.; SÁNCHEZ, J. Profit margins in Mexico's manufacturing industry. Metroeconomica, v. 51, n. 1, p. 102-121, 1996.

MANDELBAUM, K. The industrialization of Backward Areas. Oxford: Basil Blackwell, 1945. 
Julio López G.

PREBISCH, R. (1951). Problemas teóricos y prácticos del crecimiento Económico. In: GURRIERI, Adolfo (Ed.). La obra de Prebisch en la CEPAL. México: Fondo de Cultura Económica, 1982.

Obras, 1919-1948. Buenos Aires: Fundación Raúl Prebisch, 1991-1993.

ROSENSTEIN-RODAN, P. Problems of industrialization of Eastern and South-Eastern Europe. The Economic Journal, v. 53, n. 210, p. 202-211, 1943.

SCHEJTMAN, A. Economía campesina y agricultura empresarial. México: Editorial Siglo 21, 1982.

SCHYDLOWSKY, D. From import substitution to export promotion for semi-grown up industries: a policy proposal. Journal of Development Studies, v. III, n. 4, Jul. 1967.

STEINDL, J. Relaxing the international constraint on full employment. A comment. Banca Nazionale del Lavoro Quarterly Review, Mar. 1990.

SUÁREZ, F. Dos visiones de la política económica: un debate en la historia (1946 a 1970). In: ROMERO, M. E. Historia del Pensamiento Económico de México. Ciudad de México: Ed. Trillas, 2005.

THIRLWALL, A. P. The balance of payments constraint as an explanation of international growth rate differences. Banca Nazionale del Lavoro Quarterly Review, 128, p. 45-53, 1979. 